\title{
Processos éticos em enfermagem: uma revisão integrativa de estudos documentais
}

\author{
Ethical processes in nursing: an integrative review of documental studies \\ Procesos éticos en enfermería: revisión integradora de estudios documentales
}

Recebido: 08/02/2022 | Revisado: 14/02/2022 | Aceito: 23/02/2022 | Publicado: 04/03/2022

\author{
Rafael Rodrigues Polakiewicz \\ ORCID: https://orcid.org/0000-0002-8338-8084 \\ Universidade Federal Fluminense, Brasil \\ E-mail: rafao.jus@gmail.com \\ Cláudia Mara de Melo Tavares \\ ORCID: https://orcid.org/0000-0002-8416-6272 \\ Universidade Federal Fluminense, Brasi \\ E-mail: claudiamarauff@gmail.com \\ Luciana Silvério Alleluia Higino da Silva \\ ORCID: https://orcid.org/0000-0003-2786-5680 \\ Universidade Federal Fluminense, Brasil \\ E-mail: Luciana.alleluia@gmail.com \\ Rejane Eleuterio Ferreira \\ ORCID: https://orcid.org/0000-0002-9328-174X \\ Universidade Federal do Rio de Janeiro, Brasil \\ E-mail: Rejane_eleuterio@hotmail.com \\ Caroliny dos Santos Guimarães da Fonseca \\ ORCID: https://orcid.org/0000-0002-3381-732X \\ Universidad Europea del Atlántico, Espanha \\ E-mail: enfcarolinyguimaraes@gmail.com
}

\begin{abstract}
Resumo
Introdução: Os profissionais de enfermagem devem conhecer suas instituições e as leis correspondentes de sua profissão. Sob o aspecto da fiscalização, nos âmbitos assistencial e administrativo podem ocorrer erros que culminam em danos ao paciente ou a outros profissionais. Objetivo: Analisar estudos a respeito de processos éticos nos Conselhos Regionais de Enfermagem publicados na literatura. Metodologia: Trata-se de uma revisão integrativa da literatura realizada nas bases de dados SciELO e MedLine e as demais bases encontradas na BVS. Resultados: A amostra contou com 08 artigos, cujos quais analisaram, em conjunto, 05 Unidades Federativas do país. Apesar da diferença regional, os estudos apresentaram resultados semelhantes. A maioria dos processos éticos eram direcionados aos auxiliares de enfermagem e tratava-se de erros durante a administração medicamentosa ou erros durante procedimentos de sonda. Nos processos direcionados aos enfermeiros, o panorama ético mudou para assédio moral, abandono de plantão e exercício irregular ou ilegal da profissão. Considerações Finais: O estudo do perfil dos processos éticos na Enfermagem é essencial para construir abordagens direcionadas que visem minimizar a ocorrência destes.
\end{abstract}

Palavras-chave: Código de ética; Comissão de ética; Ética; Ética em enfermagem; Processo de enfermagem.

\begin{abstract}
Introduction: Nursing professionals must know their institutions and the corresponding laws of their profession. From the inspection point of view, in the care and administrative spheres, errors can occur that culminate in damages to the patient or other professionals. Objective: To analyze studies on ethical processes in Regional Nursing Councils published in the literature. Methodology: This is an integrative literature review carried out in the SciELO and MedLine databases and the other databases found in the VHL. Results: The sample had 08 articles, which analyzed, together, 05 Federative Units of the country. Despite the regional difference, the studies showed similar results. Most ethical processes were directed at nursing assistants and were about errors during drug administration or errors during tube procedures. In the processes aimed at nurses, the ethical panorama changed to moral harassment, abandonment of duty and irregular or illegal exercise of the profession. Final Considerations: The study of the profile of ethical processes in Nursing is essential to build targeted approaches that aim to minimize the occurrence of these.
\end{abstract}

Keywords: Code of ethics; Ethics committees; Ethics; Nursing ethics; Nursing process.

\section{Resumen}

Introducción: Los profesionales de enfermería deben conocer sus instituciones y las leyes correspondientes a su profesión. Desde el punto de vista de la inspección, en el ámbito asistencial y administrativo, pueden ocurrir errores que culminen en daños al paciente oa otros profesionales. Objetivo: Analizar los estudios sobre procesos éticos en los Consejos Regionales de Enfermería publicados en la literatura. Metodología: Se trata de una revisión integrativa de la 
literatura realizada en las bases de datos SciELO y MedLine y las demás bases de datos que se encuentran en la BVS. Resultados: La muestra contó con 08 artículos, que analizaron, en conjunto, 05 Unidades de la Federación del país. A pesar de la diferencia regional, los estudios mostraron resultados similares. La mayoría de los procesos éticos estaban dirigidos a los auxiliares de enfermería y se referían a errores durante la administración de medicamentos o errores durante los procedimientos de sonda. En los procesos dirigidos a enfermeros, el panorama ético cambió para acoso moral, abandono del cargo y ejercicio irregular o ilegal de la profesión. Consideraciones Finales: El estudio del perfil de los procesos éticos en Enfermería es fundamental para construir abordajes focalizados que visan minimizar la ocurrencia de estos.

Palabras clave: Código deontológico; Comités de ética; Principio moral; Ética en enfermería; Proceso de enfermería.

\section{Introdução}

A Enfermagem é uma profissão fundamentada nas relações com o paciente, família e seu contexto, cuja qual possui três categorias profissionais, tais quais: auxiliares de enfermagem, técnicos de enfermagem e enfermeiros, sendo estes com ensino superior. Nesse sentido, a Enfermagem conta com uma ampla gama de profissionais com atribuições assistenciais e administrativas diferentes que visam o bem-estar geral do paciente (Silva et al., 2015).

O Conselho Federal de Enfermagem (COFEN) e os Conselhos Regionais de Enfermagem (COREN) são entidades profissionais responsáveis por disciplinar, orientar e fiscalizar o exercício profissional da enfermagem para garantir práticas assistenciais que sejam seguras e caminhem junto ao comportamento ético (COREN-SP, 2019). Os profissionais de enfermagem devem, portanto, conhecer suas instituições e as leis correspondentes de sua profissão (Mendonça et al., 2017).

Dentro do contexto da fiscalização, sabe-se que nos âmbitos assistencial e administrativo podem ocorrer erros que culminam em danos ao paciente ou a outros profissionais, cujos quais baseam-se nos três eixos relacionados à ética: negligência, imperícia e imprudência. A partir daí, surgiu o conceito "ocorrências éticas na enfermagem", que visa comunicar as entidades legais supracitadas a respeito de condutas e ações que violam o Código de Ética dos Profissionais de Enfermagem - CEPE (Mattozinho et al., 2021).

Após a comunicação da ocorrência, o "processo ético" é instaurado pelos conselhos e, a partir daí, de fato, ocorre a análise, averiguação e julgamento da procedência do ato, culminando (ou não) na penalidade do profissional de enfermagem. Existem diversas etapas legais a seguir a partir daí, como por exemplo, a análise documental e a escuta dos denunciados e testemunhas, de modo a tornar o julgamento imparcial e o mais preciso possível (Silva et al., 2020).

Ressalta-se que, caso haja dano ou lesão do paciente, o processo ético adquire responsabilidade civil ou penal, e o infrator poderá responder não somente pelo conselho que regula seu exercício profissional, mas como pelo próprio sistema judiciário brasileiro (Oguisso et al., 2013).

O CEPE considera, além da Declaração Universal dos Direitos Humanos, a Declaração sobre Bioética e Direitos Humanos, de 2005; o Código de Ética do Conselho Internacional de Enfermeiras, de 1953; o Código de Deontologia de Enfermagem do COFEN, de 1976; e o Código de Ética de Enfermagem de 1993, reformulado em 2017, sob a Resolução n. ${ }^{\circ}$ 564, divulgada na I Conferência Nacional de Ética na Enfermagem, realizada nos dias 07, 08 e 09 de junho de 2017 (COFEN, 2017).

O CEPE estabelece como exigência a prática das ações de enfermagem e a orientação das normativas da profissão, assegurando a assistência de forma segura. Sendo a ética essencial na prática social humana, conduzindo a um senso moral do que é certo e do que é errado no exercício profissional da enfermagem, sendo fundamental que o profissional considere a condução ética e científica. Considera-se a necessidade de observação da conduta, uma vez que os problemas éticos na enfermagem são diversos, dada a prática complexa das atividades e toda a multiplicidade da profissão (Mendonça et al., 2017).

O estudo dos processos éticos é imprescindível para a melhoria da profissão, visto que ao analisar um perfil ou detectar algum tipo de padrão, medidas educativas que visam a prevenção dessas ocorrências podem ser tomadas, através da 
educação continuada. Ademais, compreender a causa e os conflitos que provocam esse desvio ético é fundamental para a correção da conduta.

Com isso, o presente trabalho visa analisar os artigos científicos sobre os processos éticos instaurados nos conselhos de enfermagem, com o objetivo de observar a causa principal desses processos e seu desfecho.

\section{Metodologia}

Trata-se de uma revisão integrativa da literatura, de caráter descritivo e abordagem qualitativa, tendo como base as seis etapas propostas previamente na literatura: identificação do tema e seleção questão de pesquisa; estabelecimento de critérios para inclusão e exclusão de estudos; definição das informações a serem extraídas dos estudos selecionados/ categorização dos estudos; avaliação dos estudos incluídos na revisão integrativa; interpretação e apresentação dos resultados (Mendes et al., 2008). Ressalta-se que o presente trabalho seguiu as diretrizes da Rede Equator a partir do checklist Preferred Reporting Items for Systematic Reviews and Meta-Analyses - PRISMA (Moher et al., 2009). O processo de pesquisa e seleção está ilustrado na Figura 1.

Figura 1. Fluxograma PRISMA de busca e seleção dos estudos. Niterói, RJ, Brasil, 2021.

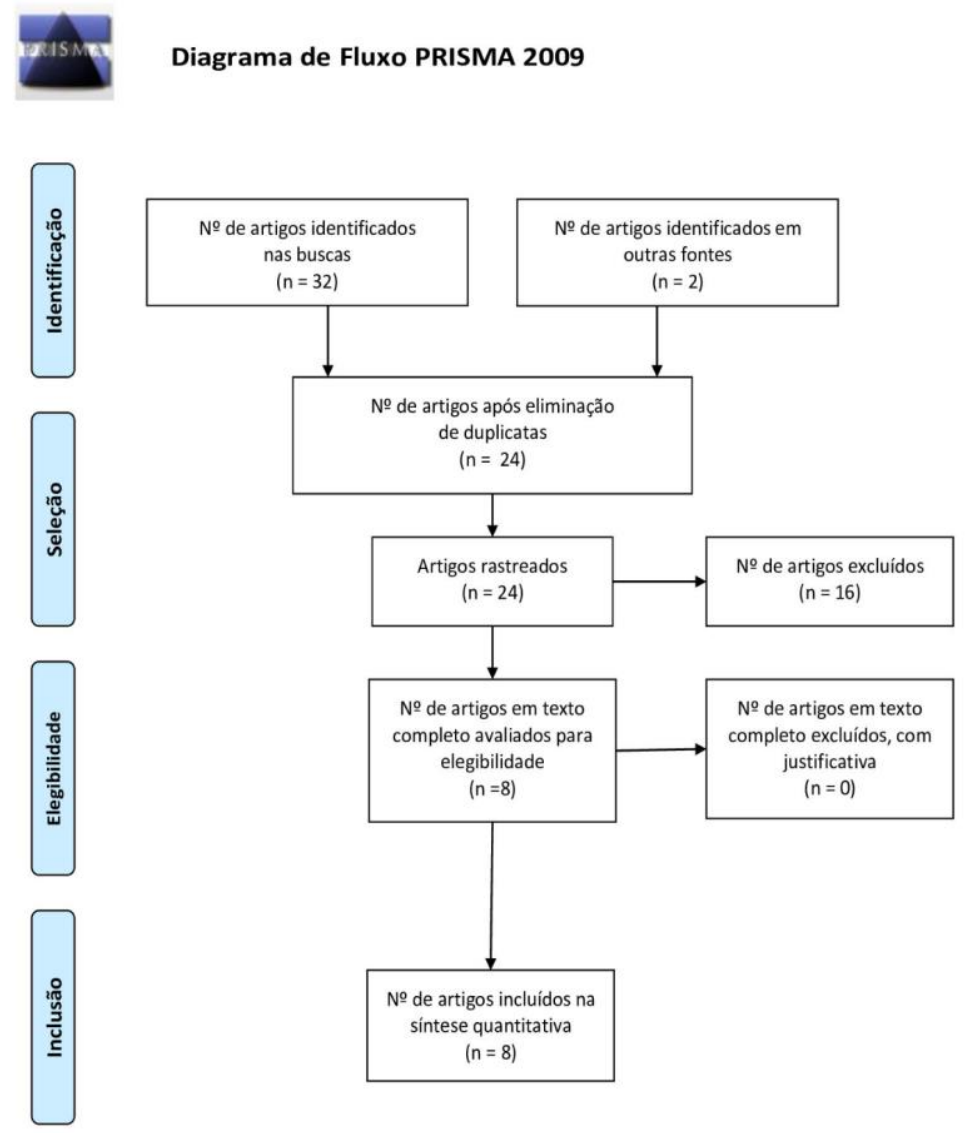

Fonte: Autores.

O presente estudo visou responder o seguinte questionamento: "Quais os tipos de processos éticos instituídos contra profissionais de enfermagem descritos na literatura?”. Para tal, foi realizada uma busca nas bases de dados Scientific Electronic 
Library Online (SciELO) e Sistema On-line de Busca e Análise de Literatura Médica (MedLine), além das demais bases indexadoras disponibilizadas na Biblioteca Virtual em Saúde (BVS).

Os descritores utilizados em pareamento com o operador booleano AND formaram a seguinte estratégia de busca: Enfermagem AND Ética Profissional AND Código de Ética AND Legislação. Os critérios de inclusão utilizados foram: artigos que trouxessem dados a respeito dos processos éticos processados nos conselhos de enfermagem; artigos em português, inglês ou espanhol. Os critérios de exclusão foram: artigos em duplicata nas bases selecionadas, estudos de revisão, editoriais e cartas ao editor. Não foi utilizado critério de ano como filtro por se tratar de um estudo sobre temática escassa na literatura a partir da análise prévia dos artigos disponíveis nas bases pesquisadas.

\section{Resultados}

Ao todo, foram encontrados 32 trabalhos, dos quais 10 estavam em duplicata nas bases de dados e somente 22 foram analisados. Após a leitura dos títulos e resumos, 08 artigos foram incluídos. Após a análise das referências desses 08 trabalhos, 02 foram incluídos por estarem enquadrados nos critérios de elegibilidade estabelecidos. Desse modo, a pesquisa contou com 08 artigos.

Todos os estudos selecionados tiveram a mesma metodologia e, portanto, tratam-se de estudos do tipo exploratóriodescritivo, documental e de abordagem quantitativa. A seguir, se explorará cada um dos estudos selecionados.

Um estudo objetivou analisar 114 registros de ocorrências éticas processadas pela Comissão de Ética de Enfermagem de um hospital. Foram analisados tanto o perfil dos profissionais quanto o caráter das ocorrências. Observou-se que os auxiliares de enfermagem foram predominantes nas ocorrências, compondo 48,25\% do total das denúncias levantadas. O número de ocorrências foi maior entre profissionais entre faixa etária de 30 a 39 anos e (55,26\%) ocorreram no período diurno (Oguisso et al., 2003).

Houve ocorrências relacionadas à imperícia, à imprudência e à negligência, porém, as duas últimas foram mais prevalentes na amostra estudada. Verificou-se que os erros mais frequentes estavam voltados para o preparo e/ou administração de medicamentos (Oguisso et al., 2003).

Sob este aspecto, um estudo objetivou caracterizar os processos éticos de enfermagem que foram processados pelo Conselho Regional de Enfermagem de Santa Catarina. Ao todo, foram analisados 128 processos (Scheneder et al., 2012).

$\mathrm{Na}$ equipe de enfermagem, os auxiliares de enfermagem foram os mais denunciados, com 66 processos, em seguida, os enfermeiros, com 61 processos, os técnicos de enfermagem com 36 processos. O estudo analisou, também, os processos envolvendo a categoria de atendente de enfermagem (07 processos) e parteiras - 02 processos (Scheneder et al., 2012).

Os processos mais frequentes giraram em torno de iatrogenias medicamentosas ou procedimentais, associadas à imperícia, à imprudência ou à negligência, totalizando 21,5\% dos processos. Em segundo lugar, veio o exercício ilegal da profissão (médico, enfermeiro ou técnico/auxiliar), com 20,9\%. Em terceiro, processos relacionados às relações interpessoais na forma de assédio (verbal, moral, física, entre outros), com 17,7\%. 14\% dos processos envolveram a responsabilidade do enfermeiro, 8,2\% agressão ao paciente, $8,2 \%$ por crimes diversos (furtos, tráfico de bebês, plágio, assédio sexual ao paciente, entre outros), $7 \%$ por negligência e 2,5\% por política profissional (Scheneder et al., 2012).

Em relação ao desfecho, a maioria dos processos (37,5\%) foram arquivados, 29,5\% levaram à advertência verbal, $15,4 \%$ à censura, $6,1 \%$ à absolvição, $6,1 \%$ à multa, 4,1\% levaram à suspensão do exercício por 29 dias e 1,3\% à cassação do direito ao exercício profissional (Scheneder et al., 2012).

Já um estudo objetivou descrever os processos éticos instaurados pelo Conselho Regional de Enfermagem de São Paulo. Ao todo, 254 processos foram analisados (Mattozinho et al., 2015). 
Dentre as categorias profissionais, os auxiliares de enfermagem foram os que tiveram maior número de ocorrências $(46,12 \%)$, seguidos pelos enfermeiros $(35,59 \%)$ e técnicos de enfermagem (18,3\%). 35,3\% dos profissionais estavam na faixa etária de 31 a 40 anos, enquanto 31,3\% entre 21 e 30 anos (Mattozinho et al., 2015).

Dentre as iatrogenias, as mais preponderantes foram as de omissão (negligência), seguidas pelas medicamentosas (imprudência ou imperícia) e iatrogenias por erro na administração da dieta - imperícia e imprudência (Mattozinho et al., 2015).

Ainda, maus-tratos ao paciente, exercício ilegal da profissão, falsificação de documento, estelionato, furto, entre outras contravenções penais tiveram a segunda maior prevalência. Ocorrências de origem de relacionamento interpessoal, abandono de plantão, inobservância do sigilo tiveram as menores prevalências nesse estudo (Mattozinho et al., 2015).

Estudo analisou 111 ocorrências no Conselho Regional de Enfermagem do Mato Grosso do Sul. A maior parte desses processos foram protagonizados por enfermeiros (68 profissionais), seguidos por técnicos em enfermagem e auxiliares - ambos com 41 profissionais (Silva et al., 2016).

A maior parte dos processos girou em torno das relações interprofissionais, como por exemplo, assédio moral e relações conflitantes. A segunda maior parte das queixas foram relacionadas à prática profissional ilegal (tanto a prática da medicina, quanto a prática da enfermagem). As iatrogenias ocuparam o terceiro lugar, dentre elas, foram iatrogenias medicamentosas ou eventos que envolveram negligência, imperícia e/ou imprudência. Processos relacionados a aborto e furto de medicamentos foram raros (Silva et al., 2016).

Pesquisa analisou 59 processos éticos do Conselho Regional de Enfermagem do Ceará. A maior parte das denúncias foram a respeito do exercício ilegal $(27,1 \%)$ e exercício irregular $(18,6 \%)$ da profissão. Houve denúncias a respeito de negligência no atendimento $(15,2 \%)$, assédio moral $(6,7 \%)$, maus-tratos a pacientes $(5 \%)$, administração incorreta de medicamentos $(3,3 \%)$. Denúncias relacionadas a: aborto, furtos, abandono de plantão, omissão de socorro, desacato, entre outras, foram minorias, com, somente, uma ocorrência de cada (Mendonça et al., 2017).

Ainda se observou que a categoria mais denunciada foi a do enfermeiro $(49,2 \%)$, seguida dos auxiliares de enfermagem $(32,2 \%)$ e dos técnicos de enfermagem (18.6\%). A respeito da penalidade (desfecho), $54.2 \%$ culminaram em advertência verbal, 30,5\% em multas, 10,2\% em absolvição, 3,4\% na cassação do direito ao exercício profissional e 1,7\% em suspensão (Mendonça et al., 2017).

Estudo analisou 18 processos éticos instaurados pelo Conselho Regional de Enfermagem do Distrito Federal para averiguar más-condutas dos profissionais de enfermagem envolvendo pacientes de 60 anos ou mais (Pereira et al., 2020).

A maior parte das denúncias atingiu os técnicos de enfermagem (57,1\%), enquanto 33,3\% atingiram os enfermeiros e 9,5\% atingiram os auxiliares. As irregularidades ocorreram na maior parte na residência do paciente $(55,5 \%)$ e as demais no âmbito hospitalar (Pereira et al., 2020).

Em relação ao tipo, a frequência de ocorrências por negligência foi a maior (27,6\%), elas envolveram situações, como: abandono de plantão, não monitorização do paciente, não administração de medicação, utilizar medicação e dieta imprópria, entre outros. Em segundo lugar, vieram as ocorrências por maus-tratos (24,1\%), como por exemplo, obrigar o idoso a lavar peças íntimas nu, cuidados inadequados após procedimentos, exploração emocional. Em terceiro, as ocorrências relacionadas à imperícia $(20,7 \%)$, como erro de medicação, troca de via de instalação de soro e dieta, infusão de dieta em acesso venoso, administrar doses inadequadas (excesso), erro durante passagem de sonda nasogástrica. Outras envolveram agressão (10,3\%), imprudência, falta de assiduidade, falsidade ideológica, furto e indisciplina, todos com 3,4\% de prevalência. Desses 18 processos, 10 culminaram em absolvição, 04 em advertência verbal, 02 em multa e 02 em arquivamento do processo (Pereira et al., 2020). 
Pesquisa analisou 34 processos éticos registrados no Conselho Regional de Enfermagem do Mato Grosso do Sul. A maioria dos profissionais envolvidos no processo ético foi da categoria de auxiliar de enfermagem, seguida pelos técnicos em enfermagem e, em menor número, enfermeiros. A maior parte dos processos éticos surgiu após infração de artigos relacionados aos deveres e responsabilidades, seguidos pelos artigos relacionados à proibição (Silva et al., 2020).

Estudo abordou os processos éticos por uma perspectiva penal, e, ao analisar 169 processos éticos do Conselho Regional de Enfermagem de São Paulo, observou-se que os processos procedimentais (97) estavam relacionados a ocorrências de negligência (40,2\%) e imperícia ou imprudência (37,1\%). Os processos de natureza atitudinal (72) mais frequentes foram a respeito de falsificação de documento, exercício ilegal, falsidade ideológica, furto e vilipêndio a cadaver. A maioria foi protagonizada por auxiliares - 49,1\% (Mattozinho et al., 2021).

\section{Discussão}

Observa-se que, na presente revisão, que a literatura disponível a respeito da ética em enfermagem é escassa, apesar de buscas em diversas bases de dados. Porém, verifica-se estudos de diferentes regiões do país, e, apesar dessa heterogeneidade, os resultados não foram tão divergentes. Nesse contexto regional, devido à escassez de estudos, somente 05 Unidades Federativas (São Paulo, Ceará, Mato Grosso do Sul, Santa Catarina e Distrito Federal) das 27 foram contempladas. Viu-se que existem estudos pioneiros desde 2003 e que, apesar de ser um tema que ganhou projeção nos últimos anos, não é recente.

A respeito do profissional, a maioria dos estudos mostrou que o auxiliar de enfermagem foi o maior alvo de processos éticos (Mattozinho et al., 2021, Silva et al., 2020, Freitas et al., 2003, Schneder et al., 2012, Mattozinho et al., 2015). Somente dois trabalhos apontam o enfermeiro como o protagonista e um trabalho observou que o técnico de enfermagem foi o profissional mais denunciado (Mendonça et al., 2017, Pereira et al., 2020, Silva et al., 2016). O que pode indicar que as infrações éticas guardam relação com a formação, ou seja, quanto maior a formação menor a infração ética.

Destaca-se que, nos estudos onde a população mais denunciada foi dos auxiliares de enfermagem, as denúncias mais prevalentes giraram em torno de iatrogenias, como por exemplo, erros durante a administração de medicamentos ou dieta, assim como erros durante procedimentos de passagem de sonda. Já nos estudos em que os enfermeiros e técnicos foram protagonistas, os processos estavam relacionados a: assédio moral, negligência (abandono de plantão e não administração de medicamentos) e exercício irregular ou ilegal da profissão enfermagem ou da medicina.

Destarte, o perfil do processo ético muda de acordo com a categoria do profissional, sendo assim, medidas educativas diferentes necessitam ser implementadas para evitar que ocorram novas situações ou comportamentos nocivos.

Uma das hipóteses na literatura para esse alto número de auxiliares envolvidos em processos éticos é que eles exercem inúmeras atividades e estão em contato, quase que, constantemente com o paciente. Ainda, o fato de que muitos auxiliares não possuem a formação necessária (carga horária limitada do curso) e que frequentemente realizam atividades de competência do técnico ou do enfermeiro, tais fatores contribuem para essa maior presença em processos éticos (Mattozinho et al., 2021).

A respeito das denúncias, pesquisa observou processos voltados para o home care, na maior parte, com ocorrências de furto e negligência. Quando se fala em home care, torna-se mais difícil ainda a fiscalização, pois há uma carência de sistema informatizado para esse tipo de serviço. Com o envelhecimento geral da população, a maior expectativa de vida e a própria situação de pandemia, é fato que estudos voltados à ética em situações de home care sejam mais frequentes e pertinentes de modo a contribuir para uma melhoria do processo de enfermagem (Pereira et al., 2020).

Outro aspecto importante relacionado às ocorrências voltadas para as relações interpessoais e interprofissionais giram em torno do gênero. Poucos estudos abordaram isso, mas a partir da perspectiva penal, estudo apontou em sua discussão a 
questão da violência obstétrica, sendo verbal e/ou física (Mattozinho et al., 2021). Os estudos analisados neste presente trabalho não analisaram a questão de assédio moral com o viés de gênero e, a partir dessa crítica, novos trabalhos podem ser realizados levando essa perspectiva em consideração.

Em relação ao desfecho dos processos, estudo observou que 54.2\% das ocorrências levaram à advertência verbal, somente uma ocorrência à suspensão por 29 dias, em 02 situações houve a cassação do direito ao exercício da profissão e 06 à absolvição (Mendonça et al., 2017). Já um estudo observou uma maior prevalência de absolvições, enquanto a medida punitiva mais frequente foi a advertência verbal, multa e arquivamento do processo ocorreram em 02 casos, respectivamente (Pereira $e t$ al. 2020). Tais dados foram de acordo com resultados apresentados em outra pesquisa (Silva et al., 2020). Em contraste, pesquisa constatou um número maior de punições do que absolvições, porém, de forma semelhante aos demais estudos, apontou que as advertências foram as punições mais prevalentes (Mattozinho et al., 2021).

Essa similaridade entre os estudos demonstra um dos pontos fortes da padronização das punições de acordo com a gravidade da infração cometida, ressaltando a importância dos conselhos de enfermagem regularem e fiscalizarem o descumprimento dos princípios éticos, porém, não deve-se esquecer que cabe ao próprio profissional da equipe de enfermagem denunciar o descumprimento das normas do CEPE (Costa et al., 2014).

Apesar disso, a própria fiscalização do exercício profissional, por ser um tema complexo e que depende de inúmeras variáveis, é bastante dificultada. Um estudo demonstrou, após entrevistas com fiscais do COREN, que a fiscalização é comprometida por fatores, como: falta de padronização na fiscalização (diferenças na fiscalização de acordo com a cidade, por exemplo), ausência de um sistema informatizado (o que daria acesso instantâneo para o fiscal), a ausência de disseminação das resoluções ou normas do exercício profissional, a lentidão no processo burocrático, entre outros (Silveira et al., 2014).

O propósito da fiscalização não é puramente punitivo, há, dentro dele, um contexto educativo que vai de acordo com os princípios do Direito Administrativo Brasileiro e da Legislação Ética. Esse contexto educativo visa educar as pessoas envolvidas nos processos éticos, além de combater desrespeito às leis que permeiam e fundamentam a prática profissional. Nesse sentido, a fiscalização por si só já é um mecanismo que visa reduzir a incidência dos processos éticos (Costa et al., 2014).

É fato que as medidas educativas são essenciais para evitar que grande parte das ocorrências aconteça. Pondo em perspectiva, os achados desse presente trabalho, no contexto das iatrogenias, a educação continuada é de extrema importância. A medida que o profissional é deslocado de setor para setor, é notório que sua rotina mudará, os aspectos práticos e administrativos mudarão. Nesse sentido, a educação continuada é fundamental para sempre manter o profissional alerta a respeito de procedimentos básicos de diversas áreas, portanto, é notório que este tipo de medida auxiliaria na redução de ocorrências relacionadas às iatrogenias procedimentais (Silva et al., 2020, Freitas et al., 2006).

Em relação às ocorrências de relacionamento interpessoal, este tipo de barreira é encontrado não somente na Enfermagem, mas em qualquer área que apresente uma espécie de hierarquia. Na verdade, lidar com esta situação requer atitudes da chefia, estimulando atividades em grupo, como rounds, dinâmicas de grupo e estratégias que visem dar credibilidade ao profissional da equipe, demonstrando que o que ele faz é parte de uma cadeia de cuidados para com o paciente, em especial, aqueles internados em unidades de cuidado intensivo (Hendricks et al., 2017, Noce et al., 2020).

\section{Considerações Finais}

O presente estudo evidenciou que há escassez na literatura a respeito da análise dos processos éticos instaurados nos Conselhos de Enfermagem, e os que foram analisados nesta revisão contemplaram poucas Unidades Federativas. Apesar disso, houve certa homogeneidade em relação ao perfil dos processos. 
Esta homogeneidade pôde ser notada pelo fato de que o auxiliar de enfermagem foi o profissional de enfermagem mais acusado de infrações éticas na maioria dos estudos; os processos envolvendo erro durante administração de medicamento ou passagem de sondas foram os mais frequentes, e; a maioria dos processos foram arquivados ou o acusado sofreu advertência verbal. Destaca-se que o perfil dos processos éticos muda de acordo com a classe profissional afetada, e, portanto, são necessários mais estudos para que possam ser elaboradas medidas de modo a reduzir a ocorrência dessas infrações éticas.

Com base nos achados, tais medidas podem ser, por exemplo, programas de educação continuada dos profissionais nos casos de erros durante administrações medicamentosas e dinâmicas de grupo em casos de assédio moral e dificuldade de relações interpessoais.

A presente revisão encontrou-se limitada por fatores relacionados à escassez de artigos sobre o tema no país e pela heterogeneidade em relação á forma de análise dos processos éticos nos estudos incluídos.

\section{Referências}

Conselho Federal de Enfermagem. (2017). Resolução n. ${ }^{\circ}$ 564, de 18 de julho de 2017. Aprova a reformulação do código de ética dos profissionais de enfermagem. COFEN.

Conselho Regional de Enfermagem de São Paulo. (2019). Manual das Comissões de Ética de Enfermagem do Estado de São Paulo. (4a ed.), Conselho Regional de Enfermagem de São Paulo.

Costa, E. D. O., Germano, R. M., \& Medeiros, S. (2014). The fiscalization of the professional practice in the Nursing Federal Council. Revista Mineira de Enfermagem,18. 10.5935/1415-2762.20140016

Freitas, G. F., \& Oguisso, T. (2003). Ocorrências éticas na enfermagem. Revista Brasileira de Enfermagem, 56:637-9. 10.1590/\$0034-71672003000600009

Freitas, G. F., Oguisso, T., \& Merighi, M. A. B. (2006). Ocorrências éticas de enfermagem: cotidiano de enfermeiros gerentes e membros da comissão de ética de enfermagem. Revista Latino-Americana de Enfermagem, 14:497-502. https://www.redalyc.org/articulo.oa?id=281421863005

Hendricks, S., LaMothe, V. J., Kara, A., \& Miller, J. (2017). Facilitators and barriers for interprofessional rounding: a qualitative study. Clinical Nurse Specialist, 31:219-28. 10.1097/NUR.0000000000000310

Mattozinho, F. C. B., \& Freitas, G. F. (2015). Ocorrências éticas de enfermagem no Estado de São Paulo: descrição fática. Acta Paulista de Enfermagem, 28:593-600. 10.1590/1982-0194201500097

Mattozinho, F. C. B., \& Freitas, G. F. (2021). Tipos penais no exercício profissional de enfermagem: abordagem quantitativa. Acta Paulista de Enfermagem, 34. 10.37689/acta-ape/2021AO00221

Mendes, K., Silveira, R., \& Galvão, C. (2008). Revisão integrativa: método de pesquisa para a incorporação de evidências na saúde e na enfermagem. Texto Contexto Enfermagem, 17:758-64. 10.1590/S0104-07072008000400018

Mendonça, F. A. C., Menezes, M. V., Amorim, S.C., Morais, F. D. M., Feitosa, E. M. N., \& Lacerda, C. M. M. (2017). Processo ético de enfermagem no estado do Ceará: reflexão para a prática profissional. Revista Enfermagem em Foco, 8:77-81. 10.21675/2357-707X.2017.v8.n2.735

Moher, D., Liberati, A., Tetzlaff, J., \& Altman, D. G. (2009). The PRISMA Group. Preferred Reporting Items for Systematic Reviews and Meta-Analyses: The PRISMA Statement. PLoS Medical, 6:e1000097. 10.1371/journal.pmed.1000097

Noce, L. G. A., Oliveira, T.S., Melo, L.C., Silva, K. F. B., Parreira, B. D. M., \& Goulart, B. F. (2020). Interprofessional relationships of a patient assistance team in critical care. Revista Brasileira de Enfermagem, 73:e20190420. 10.1590/0034-7167-2019-0420

Oguisso, T., \& Schmidt, M.J. (2013). O exercício da enfermagem: uma abordagem ético-legal. (3a ed.), Guanabara Koogan.

Pereira, M. C., Sousa, T. V., Moraes Filho, I. M., Guilhem, D. B., Lemes, J. S., \& Oliveira, M. L. C. (2020). Infrações éticas no cuidado de enfermagem à pessoa idosa. Revista Enfermagem em Foco, 11:120-5. 10.21675/2357-707X.2020.v11.n6.3911

Schneder, D. G., \& Ramos, F. R. S. (2012). Processos éticos de enfermagem no Estado de Santa Catarina: caracterização de elementos fáticos. Revista LatinoAmericana de Enfermagem, 20:Tela 1-Tela 9. 10.1590/S0104-11692012000400015

Silva, A. L. N. V., Candino, M. C. F. S., Duarte, S. J. H., \& Santos, R. M. (2016). Complaints filed against nursing professionals. Nursing Ethics, $23: 889-901$. $10.1177 / 0969733015587777$

Silva, A. L. N. V., Candido, M. C. F. S., Duarte, S. J. H., \& Santos, R. M. (2015). Infrações e ocorrências éticas cometidas pelos profissionais de enfermagem: revisão integrativa. Revista de Enfermagem da UFPE on line, 9:201-11. 10.5205/reuol.6817-60679-1-ED.0901201528

Silva, A. L. N. V., Duarte, S. J. H., Candido, M. C. F. S., Mendez, R. D. R., Machado, R. M., \& Santos, R. M., et al. (2020). Caracterização de Processos Éticos instaurados contra Profissionais de Enfermagem. Revista Nursing 23:3698-704.

Silveira, L. R., \& Ramos, S. R. F. (2014). Os maiores desafios e dificuldades para realizar o trabalho de fiscal do COREN/SC. Revista Enfermagem em Foco, 5:33-6. 10.21675/2357-707X.2014.v5.n1/2.602 
Research, Society and Development, v. 11, n. 3, e48311326711, 2022 (CC BY 4.0) | ISSN 2525-3409 | DOI: http://dx.doi.org/10.33448/rsd-v11i3.26711 